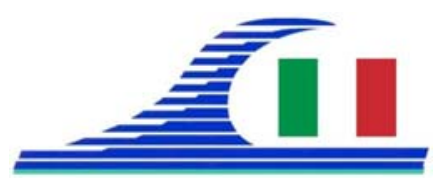

Conférence Méditerranéenne Côtière et Maritime EDITION 3, FERRARA, ITALIA (2015)

Coastal and Maritime Mediterranean Conference

Disponible en ligne - http://www.paralia.fr - Available online

\title{
Résistance au cisaillement des sédiments Utilisation du T-bar en laboratoire et effet du diamètre
}

\section{Daniel LEVACHER ${ }^{1}$, Andry RAZAKAMANANTSOA ${ }^{2}$, Ritesh GUPTA ${ }^{1,2,3}$}

1. Université de Normandie, UniCaen, Laboratoire Morphodynamique Continentale et Côtière, UMR 6143 M2C, 24 rue des Tilleuls, 14000 Caen, France.

daniel.levacher@unicaen.fr

2. GERS Department, IFSTTAR Nantes, Route de Bouaye CS 54, F-44344 Bouguenais cedex, France. andry.rzakamanantsoa@ifsttar.fr

3. Department of Civil Engineering, National Institute of Technology, Hamirpur, 177005, India.riteshgupta.nith@gmail.com

\section{Résumé:}

La résistance au cisaillement non drainé Su des sols fins et en particulier des sédiments, est un paramètre important pour l'étude de la stabilité de talus, de la circulation des engins sur couches déposées en bassin de lagunage, du suivi de la déshydratation et du séchage naturel, de la consistance et sensibilité des sédiments. La résistance au cisaillement non drainé peut être mise en relation avec la teneur en eau et les limites d'Atterberg. Plusieurs outils sont disponibles pour sa détermination in situ et en laboratoire. Pour l'obtention d'un profil en continu des mesures de Su, les pénétromètres à écoulement - full flow penetrometer - sont utilisés en ingénierie offshore et en modélisation en centrifugeuse avec des outils miniatures. En laboratoire, à l'aide d'un outil miniature, il est nécessaire d'étudier conditions d'utilisation et de valider les mesures acquises. Un T-bar miniature (TBT) a été développé et utilisé en même temps qu'un scissomètre de laboratoire (VST) dans des sols fins reconstitués et des sédiments. Ce dernier outil ne fournit que des mesures ponctuelles et ne permet pas d'effectuer des cycles pénétration-extraction comme le T-bar permettant d'étudier la dégradation des sols. Le T-bar de laboratoire est présenté avec la justification de sa géométrie et des conditions d'utilisation vis-à-vis des recommandations établies par l'ingénierie offshore. Les sols testés et les conditions expérimentales sont brièvement décrits. Lors de premiers essais, les influences de la taille et de la rugosité du diamètre du T-bar sont observées et commentées.

Mots-clés: Essai T-bar miniature, Kaolin, Sédiment, Résistance au cisaillement non drainé, Effet d’échelle, Essai en laboratoire.

\section{Introduction}

La résistance au cisaillement non drainée Su des sols fins gouverne les mécanismes de stabilité et de rupture de bon nombre d'ouvrages en ingénierie géotechnique côtière (talus, berges, fondations, barrages en terre, ...). L'identification du paramètre Su se fait 
Côtes méditerranéennes menacées :

Risques et défis dans le contexte du changement climatique

en partie in situ pour pallier au prélèvement délicat notamment dans le cas de sols fins mous. Deux essais sont utilisés, préconisés pour des reconnaissances en grande profondeurs le CPT (Cone Penetrometer Test) et le VST (Vane Shear Test). Un autre essai très utilisé en ingénierie offshore, le TBT (T-Bar Test) a fait son apparition, il combine les avantages des deux précédents. On retrouve ces appareillages en taille réduite pour la caractérisation des sols fins modélisés en centrifugeuse géotechnique, (STEWART \& RANDOLPH, 1994). Dans les laboratoires de géotechnique pour des essais à gravité normale, seul le VST miniature est répandu. L'intérêt de miniaturiser le TBT en laboratoire est important. Il permet (i) d'identifier en continu Su sur de grandes hauteurs (carottes de sols, cellules et cuves d'essai de sols reconstitués), (ii) d'effectuer des cycles pénétration-extraction et (iii) d'appréhender la sensibilité des sols fins mous. Encore faut-il définir les limites et les performances de ce TBT de laboratoire, c'est-àdire définir les procédures d'utilisation de l'essai. C'est l'objet de cette communication. Peu d'études ont été menées en laboratoire sur l'étude des mécanismes de rupture et les différentes influences pour de faibles profondeurs inférieures à $0.50 \mathrm{~m}$ (OROZCOCALDERON et al., 2010). L'effet de la géométrie du TBT a été étudié dans des cellules d'essai de hauteur limitée à 200 mm et de diamètre 300 mm.

\section{Matériels et méthodes}

\subsection{Sol fin d'essai}

Il s’agit d'une argile communément utilisée en laboratoire, une kaolinite Speswhite $^{\mathrm{TM}}$ qui permet de reconstituer d'épaisses couches d'argile à propriétés physiques et mécaniques contrôlées dans le cas de sols argileux modèles en centrifugeuse. Le tableau 1 en rapporte les caractéristiques moyennes issues de précédentes études.

Le matériau d'essai est préparé par malaxage avec une quantité d'eau nécessaire à l'obtention d'une teneur en eau w voisine de $2 \mathrm{~W}_{\mathrm{L}}$, égale à $103 \%$ pour ces essais. Le mélange est laissé au repos pendant 24h, puis malaxé de nouveau pendant une heure avant le remplissage des cellules d'essai. Puis un premier essai est effectué au temps T0.

Tableau 1. Données relatives à la kaolinite.

\begin{tabular}{|c|c|c|c|c|c|c|c|c|c|c|}
\hline $\begin{array}{l}W_{L} \\
(\%)\end{array}$ & $\begin{array}{l}W_{P} \\
(\%)\end{array}$ & $\begin{array}{l}I_{P} \\
(\%)\end{array}$ & $\begin{array}{l}\text { Surface } \\
\text { spécifique } \\
\left(\mathrm{m}^{2} / \mathrm{g}\right)\end{array}$ & $\begin{array}{l}\rho_{s} \\
\left(\mathrm{~g} / \mathrm{cm}^{3}\right)\end{array}$ & $\begin{array}{l}\text { Particules } \\
<2 \mu m\end{array}$ & $\begin{array}{l}\text { Particules } \\
>10 \mu m\end{array}$ & $\begin{array}{l}k \\
(m / s)\end{array}$ & $\begin{array}{l}C v \\
\mathrm{~m}^{2} / \mathrm{s}\end{array}$ & $C c$ & Cs \\
\hline $55^{*}$ & $30 *$ & $25^{*}$ & 30 & 2.65 & 79 & 0.5 & 0.15 à $1010^{-9}$ & 1.5 à $710^{-7}$ & 0.5 & 0.1 \\
\hline
\end{tabular}

\subsection{Matériel d'essai}

Le TBT miniaturisé est constitué à la base d’un cylindre horizontal formant un T avec la tige verticale qui permet son installation sur un dispositif d'essai dédié à des essais de 
pénétration. Ce dernier comprend un vérin asservi en déplacement et/ou en effort. Le TBT a un corps d'épreuve en aluminium, équipé de jauges de déformation montées en pont complet ; l'ensemble est revêtu d'une résine époxy autorisant l'immersion du TBT. Le modèle utilisé pour les essais entrepris a une capacité d'effort maximal de $375 \mathrm{~N}$ en traction/compression pour une profondeur de $0.60 \mathrm{~m}$. Le dispositif d'essais de pénétration est doté de moteurs pas-à-pas pour un positionnement en $\mathrm{x}$ et en $\mathrm{y}$ (plan horizontal) et un mouvement vertical en $\mathrm{z}$ (pénétration et extraction). L’acquisition des données se fait à une fréquence de $5 \mathrm{~Hz}$.

\subsection{Etalonnages du TBT miniature}

La calibration du capteur d'effort se fait d'une manière expérimentale simple à l'aide d'un plateau-nacelle accroché par un fil au corps d'épreuve sur lequel sont disposées des masses. Le chargement et déchargement de ces masses est corrélé à la sensibilité du TBT exprimée en mV/V (CHERIFI, 2013). La vitesse de pénétration v du TBT dans les sols fins argileux doit être déterminée de telle manière à respecter les conditions d'essais en comportement non drainé. HOUSE et al. (2001) ont fourni une relation entre une vitesse de pénétration adimensionnelle $\mathrm{V}$ en fonction de la vitesse de pénétration $\mathrm{v}$, du diamètre $\mathrm{d}$ de la tige et du coefficient de consolidation $\mathrm{Cv}$ à partir d'essais dans un kaolin à des profondeurs de 2 à 10m. Les conditions non drainées s'avèrent respectées lorsque $\mathrm{V}$ a une valeur supérieure à 30 . Pour nos essais la vitesse de pénétration $\mathrm{v}$ a été prise égale à $2 \mathrm{~mm} / \mathrm{s}$ avec un $\mathrm{Cv}$ de $1.5 \times 10^{-7} \mathrm{~m}^{2} / \mathrm{s}$, un diamètre de $3 \mathrm{~mm}$ et $\mathrm{V}$ égale à 40 .

$\mathrm{V}=\mathrm{v} \times \mathrm{d} / \mathrm{Cv}$

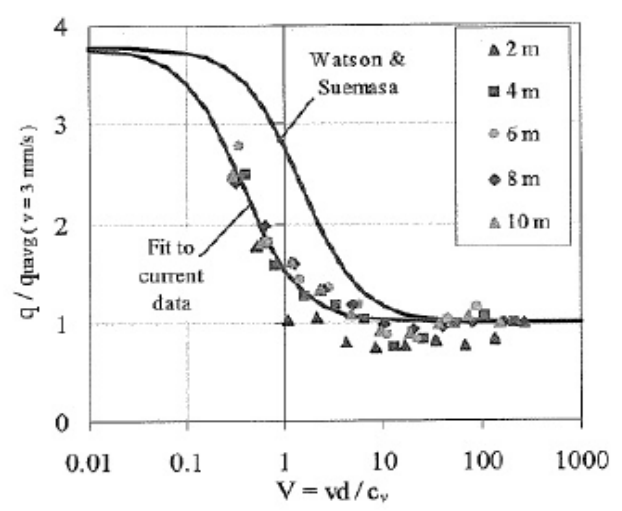

a) Vitesse d'essai (HOUSE et al.)

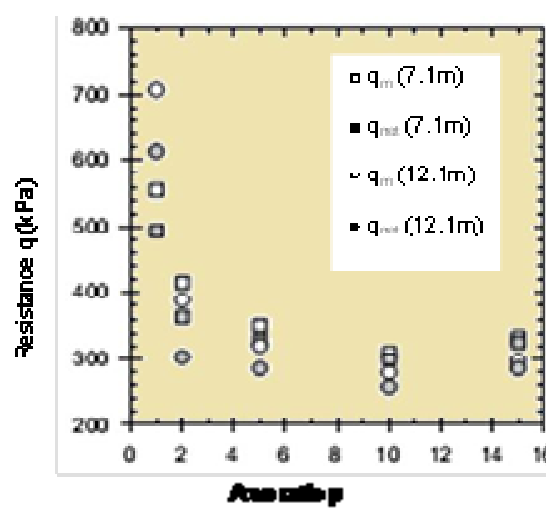

b) Effet de la tige, (YAFRATE et al., 2007)

Figure 1. Relations déduites d'essais TBT.

\subsection{Géométrie du TBT miniature}

\subsubsection{Définition des coefficients}

Les principaux rapports géométriques qui ont permis de standardiser les essais TBT in situ sont les suivants : 
Côtes méditerranéennes menacées :

Risques et défis dans le contexte du changement climatique

- élancement du barreau horizontal $\lambda=\mathrm{L}$ (longueur)/D (diamètre),

- rapport des tiges $\alpha=\mathrm{d}$ (diamètre de la tige verticale)/D (diamètre du barreau),

- rapport des sections $\mu=($ section de l'empreinte du barreau)/(section de la tige verticale).

On a la relation : $\mu=4 \mathrm{DL} /\left(\pi \mathrm{d}^{2}\right)$

Le coefficient $\lambda=4$ est suffisant pour garantir les conditions de déformations planes autour du cylindre horizontal. Dans ce cas, la relation (2) avec $\mathrm{D} / \mathrm{d}=1 / \alpha=\beta$ devient : $\mu=4 \lambda D^{2} / \pi d^{2}$ soit $\mu=4 \lambda \beta^{2} / \pi$ et finalement, $\mu=5.09 \beta^{2}$.

Le coefficient $\mu \geq 10$ est suffisant pour que la tige verticale n'ait aucune influence sur l’effort de résistance à la pénétration, voir figure (1b), (YAFRATE, 2007).

\subsubsection{Géométries proposées}

Pour les différents essais, une série de 5 barreaux ont été proposés en respectant un coefficient $\lambda$ de 4 . Ils sont acier usiné i.e. état de rugosité considéré lisse dans ce cas. La tige verticale permettant le montage de ces barreaux comme embout au corps d'épreuve est de $3 \mathrm{~mm}$ ce qui donne pour ces 5 barreaux un coefficient $\mu$ supérieur à 10, voir tableau 2. Une série de barreaux a été proposé avec un état de rugosité différent. Ils ont été rendus rugueux à l'aide d'un collage de sable fin de Fontainebleau de diamètre médian 0.2 mm sur la surface latérale de chaque barreau, voir tableau 2.

Tableau 2. Paramètres géométriques des barreaux utilisés.

\begin{tabular}{|c|c|c|c|c|c|c|c|}
\hline \multicolumn{6}{|c|}{ Caractéristiques géométriques des TBT utilisés } & \multirow[t]{2}{*}{ T-bar lisse } & \multirow[t]{2}{*}{ T-bar rugueux } \\
\hline$D(\mathrm{~mm})$ & $L(\mathrm{~mm})$ & $\lambda$ & $d(\mathrm{~mm})$ & $\beta$ & $\mu$ & & \\
\hline 5 & 20 & 4 & 3 & 1.67 & 14.13 & & \\
\hline 7.5 & 30 & 4 & 3 & 2.50 & 31.81 & $=$ & \\
\hline 10 & 40 & 4 & 3 & 3.33 & 56.44 & & \\
\hline 12.5 & 50 & 4 & 3 & 4.17 & 88.08 & & \\
\hline 15 & 60 & 4 & 3 & 5.00 & 127.25 & & \\
\hline
\end{tabular}

\subsection{Détermination de Su}

La force de pénétration ou d'extraction Fv est enregistrée au cours de chaque essai. La détermination de Su se fait à l'aide d'un facteur de portance $\mathrm{N}_{\mathrm{TBT}}$ selon l'état de rugosité, lisse ou rugueux, selon la relation (3). L’effort de pénétration et/ou d'extraction est rapporté par unité de surface (empreinte du barreau) et divisé par le facteur $\mathrm{N}_{\text {TBT. }}$. Des simulations numériques en déformations planes ont permis de proposer des facteurs couramment utilisés, voir tableau 3.

$\mathrm{Su}=\mathrm{Fv} /\left(\mathrm{D} \times \mathrm{L} \times \mathrm{N}_{\mathrm{TBT}}\right)$ 
Tableau 3. Facteurs $N_{\text {Твт }}$ recommandés.

\begin{tabular}{ll}
\hline Etat de surface & Facteur $\boldsymbol{N}_{\text {TB }}$ \\
\hline Lisse $S$ & 9.2 \\
Rugueux $R$ & 12 \\
Etat indéterminé $S<$ état $<R$ & 10.5 \\
\hline
\end{tabular}

\subsection{Cellule d'essai}

Des cellules d'essais cylindriques (diamètre $300 \mathrm{~mm}$, hauteur $205 \mathrm{~mm}$, épaisseur de paroi $10 \mathrm{~mm}$ ) munies à la base d'un système de drainage avec récupération de l'eau au cours du temps par flaconnage en plastique étanche, ont servi à tous les essais, voir figure 2 .

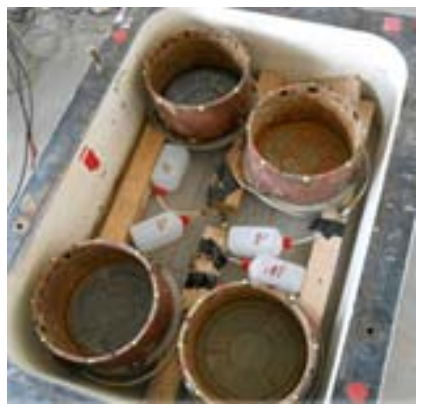

a) Cellules et flacons.

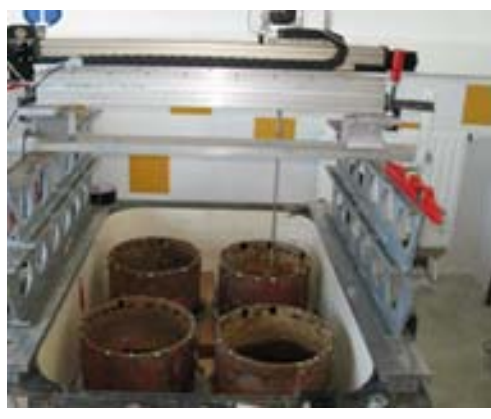

b) Appareillage T-bar.

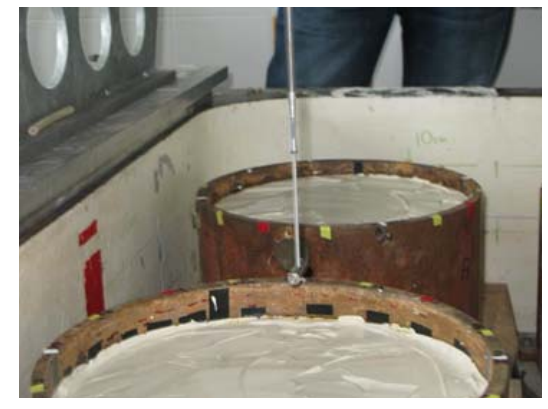

c) T-bar avant pénétration.

Figure 2. Dispositif expérimental pour les essais de T-bar.

\section{Effet du diamètre du T-bar miniature lisse et rugueux}

A partir des essais pénétration-extraction, effectués au cours du temps (T0 étant le temps de référence à $24 \mathrm{~h}$ après le remplissage), des mesures de teneurs en eau, il a été possible de suivre l'évolution de Su et WC en fonction du temps et ce, en fonction de différents diamètres du T-bar et de leur état de rugosité. La cohésion non drainée augmente avec le temps en relation avec la teneur en eau et en profondeur respectant le type de drainage appliqué à l'échantillon en surface et au fond (LEVACHER et al., 2014). On observe des différences entre les valeurs de Su pour les 2 états de rugosité testés. La variation de Su en fonction du diamètre, quel que soit l'état de rugosité est montrée sur la figure 3. On observe une tendance identique à celle observée par YAFRATE et al. (2007). Les valeurs de Su semblent se stabiliser à partir d'un diamètre de 8 voire $10 \mathrm{~mm}$. Elles sont aussi plus dispersées pour les diamètres lisses, figure 3a.

\section{Conclusions}

Le T-bar parait être un outil de laboratoire pertinent pour la détermination de Su mais de nombreuses questions sur la procédure (conditions) d'essai restent posées. Dans le cadre de ces essais, ont été caractérisés les effets de la taille du diamètre $\mathrm{D}$, de l'état de 
Côtes méditerranéennes menacées :

Risques et défis dans le contexte du changement climatique

surface du T-bar, de la proximité entre essais et de l'effet de bord de la cellule. On note qu'au-delà d'un diamètre $10 \mathrm{~mm}$, avec $\lambda=4$ et $\beta=3.33$, les valeurs de Su se stabilisent montrant ainsi que l'effet du diamètre $d$ devient négligeable. Cette condition ne reste valable que pour des conditions d'écoulement du sol autour du T-bar bien établies ; ceci dépend des valeurs de Su. On peut ajouter que les effets de bord et de proximité entre essais deviennent négligeables à partir d'une distance de 2à $3 \mathrm{D}$.

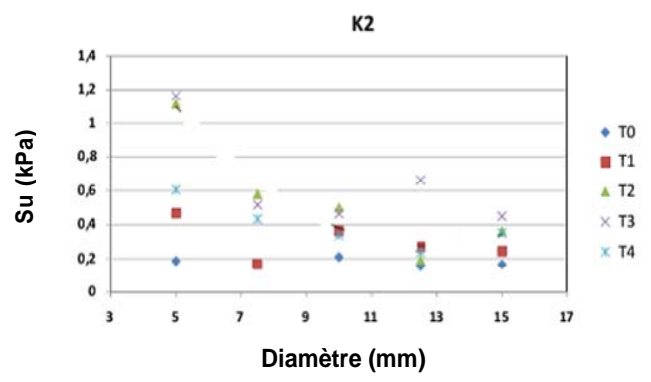

a) T-bar lisse.

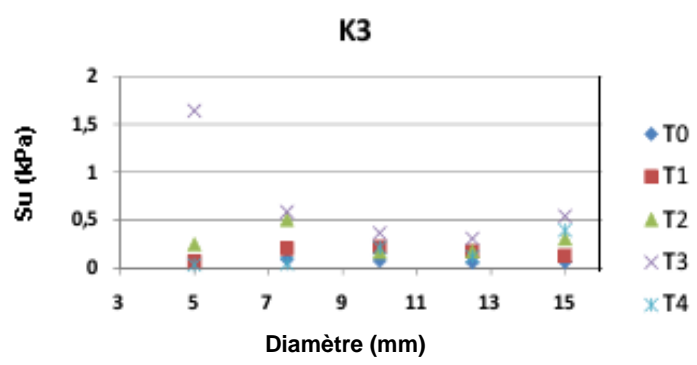

b) T-bar rugueux.

Figure 3. Effet de la taille du diamètre D du T-bar sur la mesure de Su. Les essais sont espacés de 7 jours de T0 à T4, (CHERIFI, 2013).

\section{Références bibliographiques}

BOUSSAID K. (2005). Sols intermédiaires pour la modélisation physique: application aux fondations superficielles. Thèse de doctorat, Ecole Centrale de Nantes, Université de Nantes, 287 p.

CHERIFI E. (2013). T-bar laboratory testing on reconstituted clay. Master report, Caen University, $65 \mathrm{p}$.

HOUSE A.R., OLIVEIRA J.R.M., RANDOLPH M.F. (2001). Evaluating the coefficient of consolidation using penetration tests. International Journal of Physical Modelling in Geotechnics, Vol. 1, pp 17-26.

LEVACHER D., RAZAKAMANANTSOA A., KIMURA F., KATSUMI T. (2014). Use of sedi-bar test for the characterization of soft soils and sediments. GeoEnvironmental Engineering, GEE 2014, Sapporo, Japan, May 30-31, pp 8-15.

OROZCO-CALDERON M., FORAY P., PUECH A., HALL S. (2010). Essais au miniTbar à faible penetration. Journées Nationales de Géotechnique et de Géologie de l’Ingénieur JNGG2010, Grenoble, pp 135-142.

STEWART D.P., RANDOLPH M.F. (1994). T-bar penetration testing in soft clay. Journal of Geotechnical Engineering, Vol. 120, pp 2230-2235. http://dx.doi.org/10.1061/(ASCE)0733-9410(1994)120:12(2230)

YAFRATE N.J., DE JONG J.T., DE GROOT D.J. (2007). The influence of full-flow penetrometer area ratio on penetration resistance and undrained and remoulded shear strength. Offshore Site Investigation and Geotechnics, Confronting New Challenges and Sharing Knowledge, London, pp 461-468. 